\title{
Collectif, Romance Rewritten: The Evolution of Middle English Romance. A Tribute to Helen Cooper
}

\section{Martine Yvernault}

\section{(2) OpenEdition}

1 Journals

Édition électronique

URL : https://journals.openedition.org/ccm/7563

DOI : $10.4000 / \mathrm{ccm} .7563$

ISSN : 2119-1026

Éditeur

Centre d'études supérieures de civilisation médiévale/Université de Poitiers

\section{Édition imprimée}

Date de publication : 1 juin 2021

Pagination : 186-189

ISBN : 978-2-490783-09-0

ISSN : 0007-9731

\section{Référence électronique}

Martine Yvernault, "Collectif, Romance Rewritten: The Evolution of Middle English Romance. A Tribute to Helen Cooper », Cahiers de civilisation médiévale [En ligne], 254 | 2021, mis en ligne le 01 juin 2021,

consulté le 25 novembre 2022. URL : http://journals.openedition.org/ccm/7563 ; DOI : https://doi.org/ $10.4000 / \mathrm{ccm} .7563$

\section{(c) $\underset{\mathrm{EY}}{(\mathrm{NQ})} \mathrm{\Theta D}$}

Creative Commons - Attribution - Pas d'Utilisation Commerciale - Pas de Modification 4.0 International - CC BY-NC-ND 4.0

https://creativecommons.org/licenses/by-nc-nd/4.0/ 
Romance Rewritten: The Evolution of Middle English Romance. A Tribute to Helen Cooper, Elizabeth ARCHIBALD, Megan G. Leitch, Corinne J. SAunders (dir.), Cambridge, D. S. Brewer (Studies in Medieval Romance, 22), 2018.

L'ouvrage de 295 p., bibliographie et index compris, est un volume d'hommages en l'honneur d'Helen Cooper dont la carrière se partagea entre les universités d'Oxford et de Cambridge, au Magdalene College (dont elle est Professeur émérite) et où elle occupa la chaire de littérature médiévale et Renaissance anglaise. L'ouvrage qui lui est dédié traduit l'intérêt d'H. Cooper pour la romance, genre auquel elle consacra de nombreuses études, notamment The English Romance in Time: Transforming Motifs from Geoffrey of Monmouth to the Death of Shakespeare, publiée en 2004 (Oxford, Oxford University Press).
Paradoxalement, l'introduction de Megan Leitch cible en premier lieu non pas le roman mais la parodie du genre telle qu'elle fut expérimentée, par ex., par Chaucer dans l'écriture du Conte de Sir Thopas, parodie qui témoigne de tout un héritage littéraire. Il s'agit en fait de distinguer le roman populaire et le roman courtois, et de redorer le blason du roman populaire en rappelant qu'il avait un auditoire et un lectorat aux XIV et XV $\mathrm{s}$. Ce volume collectif propose donc une vision large du corpus des romans, comprenant non seulement les textes s'adressant à un public averti et savant, mais aussi les romans populaires anonymes anglais ainsi que leurs sources textuelles continentales. Les contributeurs soulignent ainsi la 
«nature protéiforme du genre » (p. 3) et ils explorent la question de son évolution, des réécritures, de sa réception, de l'utilisation des conventions et motifs ou, au contraire, des distances prises par les auteurs. En insistant ainsi sur la question de la réécriture et de l'adaptation, l'ouvrage met en relief la centralité des « memes » dans l'analyse des romans menée par H. Cooper, ces formes et motifs conventionnels qui, presque de manière génétique, se transmettent, se reproduisent, s'adaptent, prennent divers aspects dans l'écriture, du Moyen Âge à l'époque pré-moderne et qui, loin de s'inscrire dans les catégories délimitées que l'on a nommées matières (matière de Rome, de Bretagne...), entrent en résonance.

Neil Cartlidge ouvre la première partie, qui porte sur les discordances dans l'écriture du roman. L'a. part du postulat de Northrop Frye, énoncé dans son Anatomy of Criticism, selon lequel le roman médiéval construit un monde fondé sur le bien et le mal reflétant souvent tout un monde intérieur, des sens plus profonds, les pulsions sourdes et latentes des auteurs et lecteurs. À cela s'ajoutent les liens qui sont souvent établis avec le conte de fées, notamment pour Erich Auerbach. Cette approche, selon N. Cartlidge, tend à dévaluer la spécificité textuelle du roman, voire sa littéralité, ses spécificités lexicales, la réalité du contexte historique, pour seulement privilégier sa profondeur abordée au prisme de différentes interprétations, par ex. psychanalytique chez Bruno Bettelheim. S'opposent ainsi la fabrique du texte et son herméneutique, au détriment de régimes moins nobles et moins intellectuels comme le comique que l'on trouve dans des textes tels que Sir Tristrem, Sir Thopas, à certains endroits de Lybeaus Desconus, improprement définis comme des parodies selon N. Cartlidge, qui les lit comme des formes de dissonance pleinement assumées par leurs auteurs.

L'étude de Marcel Élias poursuit l'exploration des formes anomiques du roman en ciblant trois motifs qui correspondent à trois types de personnages : le héros ambivalent, le noble maure, le challenger menaçant (que l'on trouve dans Sir Gawain and the Green Knight, par ex.). Ces trois figures dissonantes expriment, selon l'a., le déclin des idéaux chevaleresques ainsi que l'extension du pouvoir ottoman dans l'Europe du $\mathrm{XIV}^{\mathrm{e}} \mathrm{s}$. tandis que la chrétienté vacille en Terre sainte. Ces trois motifs sont des exemples de réécriture de textes en ancien français et de chansons de geste anglo-normandes. L'adaptation reflète non seulement l'emprunt de personnages mais aussi le passage de la chanson de geste au roman grâce aux motifs importés et visibles, par ex., dans The Sultan of Babylon, Duke Roland and Sir Otuel of Spain, ou dans le roman Otuel. Ces dissonances ne s'inscrivent pas seulement dans une perspective littéraire mais rendent compte des questions théologiques et morales qui agitent le XIv ${ }^{\mathrm{e}} \mathrm{s}$. (le rapport de l'homme à Dieu, l'immoralité de la guerre telle que Saint Augustin la dénonce dans la Cité de Dieu).

Faisant écho aux réflexions de N. Cartlidge sur le mode comique qui construit aussi le roman médiéval, Christopher Cannon observe l'inversion des idéaux chevaleresques, l'humour et les formes du rire dans Malory, pour qui l'intrusion de la comédie met au jour les failles qui auront raison de la solidité de la communauté de la Table ronde. Chez Malory, selon C. Cannon, le comique réside dans les contradictions qui sapent la Table ronde autant qu'elles en assurent le fondement. Ainsi l'infidélité de Guenièvre bafoue son époux Arthur, mais Lancelot demeure le soutien indispensable du roi. Plus précisément, le Morte Darthur autorise le comique jusqu'au moment où la communauté arthurienne bascule dans la tragédie et périt. Le comique ne se conçoit donc pas sans la prise en compte de la tragédie; le mode comique est didactique et possède une raison d'être éthique dans la mesure où il permet de percevoir faille, corruption, contradiction.

À travers ces trois études, l'ouvrage met l'accent sur la signification des formes de dysfonctionnement dans le roman. La seconde partie envisage la spécificité textuelle du roman, déjà commentée par N. Cartlidge, mais en se penchant sur les stratégies narratives dans les romans ou les textes assimilés au roman.

Jill Mann observe le début du Conte du Chevalier, qui relate en fait la fin de la guerre de Thésée contre les amazones et son mariage avec la reine Hippolyte. Le choix de commencer par la narration d'une fin pose le problème du début d'une autre histoire. Une histoire ancrée dans un passé plus ou moins ancien ou mythique doit forcément arrêter un moment précis à partir duquel une autre histoire débute. Faisant de la fin non pas une clôture mais une perspective propice à une autre narration, le roman renforce la nature ouverte et dynamique de sa substance même - l'aventure - dont la fin n'est jamais réellement un point final. De même, dans la trame centrale d'une histoire, des fils narratifs secondaires peuvent venir se 
glisser, qu'ils deviennent des histoires à part entière ou restent à l'état d'allusions. La reprise narrative - son rebond - se marque par l'opposition entre la temporalité stagnante et les indications stylistiques informant sur la rupture de la latence. Reprenant les considérations métaphysiques du discours de Thésée, Jill Mann avance, à juste titre, que ces traitements de la fin et du début dans le roman ne font que refléter le grand texte de la vie humaine avec ses arrêts plus ou moins définitifs, ses naissances, ses sursauts, ses permanences et impermanences, et font écho au traitement des cassures et contradictions du roman traitées dans la première partie.

C'est à la visée politique, sous-jacente dans l'écriture romanesque, que Robert F. Yeager s'intéresse. L'a. explore les motivations de Gower dans le $8^{\mathrm{e}}$ livre de sa Confessio Amantis, Apollonius de Tyr, dont l'inscription dans le genre du roman n'est pas ferme. Sa place dans la Confessio est difficile à justifier sauf si l'on considère que Richard II fut le destinataire de la Confessio. Derrière le choix de la narration d'histoires et d'aventures, on discerne un discours sur la responsabilité politique du monarque. Plus qu'un roman, un genre que Gower ne prisait guère, Apollonius de Tyr serait donc un Bildungsroman.

Dans le sillage de James Wimsatt en particulier, Elizabeth Archibald s'attache à montrer que Malory eut accès à la totalité des textes arthuriens français et se les appropria notamment en donnant de l'ampleur à la figure d'Arthur et en soulignant les vertus chevaleresques de Lancelot en dépit de son rôle dans le déclin du monde arthurien. Son étude met en regard, en s'appuyant dans le détail sur des passages bien ciblés de Malory, le corpus défini comme le cycle Post-Vulgate (Roman du Graal) et celui qui est dénommé cycle de la Vulgate. À travers cette approche comparative, montrant les partis pris, les altérations, les amplifications de Malory dans son Morte Darthur, l'a. livre une abondante bibliographie (incluant notamment les études de Edward Donald Kennedy, de Fanni Bogdanow, d'H. Cooper, de Richard Trachsler).

S'inspirant d'une étude d'H. Cooper, « Afterword: Malory's Enigmatic Smiles " dans Emotions in Medieval Arthurian Literature: Body, Mind, Voice, ouvrage édité par Franck P. Brandsma, Carolyne Larrington et Corinne J. Saunders (Cambridge, D. S. Brewer [Arthurian Studies, 83], 2015), Barry Windeatt explore une forme de narration particulière dans le roman. Il interroge le langage du corps, l'implicite, le codage des émotions, le signe et la constitution d'un lexique approprié à ces domaines (gestural lexicon). Ce lexique particulier participe à la narration tout en renseignant sur la valeur et l'expression des émotions au Moyen Âge. Sont ainsi déchiffrées des expressions et attitudes corporelles très variées (rougir, s'évanouir, rester immobile, regarder, rire, pleurer, etc.) dans les romans, mais aussi dans les lais bretons tels que Le Freine, Launfal, Degaré, Sir Orfeo.

La troisième partie concerne un aspect essentiel des romans : la spiritualité.

C'est sur Sir Amadace, Sir Launfal, et surtout Sir Cleges que s'appuie Marco Nievergelt afin de souligner l'articulation - a priori contradictoire entre le spirituel et le matériel, le salut et les sujets économiques. Pourtant le paradoxe s'atténue si l'on recentre le propos sur des concepts économiques spécifiques qui sont diversement valorisés et érigés en vertus dès lors qu'ils s'inscrivent dans une perspective théologique (par ex., l'échange, la générosité, le don, la charité). Une lecture théologique de ces concepts économiques nuance, par conséquent, les contradictions apparentes qui font partie d'une mise en œuvre stylistique destinée, dans Sir Cleges, à mettre en évidence le paradigme de toute forme de don : l'incarnation.

Miriam Edlich-Muth observe 1'adaptation d'un " meme », l'histoire des enfants cygnes, dont le traitement dans Dolopathos au XII ${ }^{\mathrm{e}}$ s., La Naissance $d u$ Chevalier au Cygne et enfin Chevalere Assigne au XIV $^{\mathrm{e}} \mathrm{s}$. reflète des reprises et des divergences montrant que le contenu didactique chrétien, accordant une place essentielle à la providence divine, dilue les composants magiques et surnaturels des textes sources.

Reflétant les courants de la recherche contemporaine, qui associent humanités, sciences sociales, sciences cognitives, et rappelant également l'ouvrage récent Cognitive Confusions: Dreams, Delusions and Illusions in Early Modern Culture (Ita MAC CARTHY, Kirsti Sellevold et Olivia Smith [dir.], Cambridge, Legenda, 2016), Corinne Saunders expose le Morte Darthur au même prisme contemporain d'analyse en ciblant la voix, l'écoute, la vue, et ce que ces expériences sensorielles nous dévoilent du monde intérieur du récepteur, des communautés médiévales, et des 
cultures et lecteurs contemporains. De telles orientations scientifiques montrent que l'approche d'un objet littéraire ou esthétique dépend de la mise en relation d'expériences et de faits extérieurs à l'individu et de leur intériorisation. L'approche n'est cependant pas nouvelle puisque la traduction intérieure des perceptions extérieures fut commentée par Aristote, Avicenne, Saint Augustin. C. Saunders démontre qu'au-delà de sa prédilection pour la description de faits et d'actions, le Morte Darthur se préoccupe aussi du monde intérieur, du rôle joué par la perception sensible et imaginaire, et interroge la frontière qui sépare - parfois de manière ténue - le monde terrestre et le monde métaphysique, l'individu et le divin, l'action humaine et la manière dont la providence et la grâce, mais aussi les visions et les manifestations prophétiques, peuvent agir sur les actes et le destin des hommes.

La quatrième et dernière partie porte sur les reprises tardives du genre romanesque à la fin $\mathrm{du}$ $\mathrm{XV}^{\mathrm{e}}$ s., au XVI s. et du XVIII ${ }^{\mathrm{e}}$ au $\mathrm{XX}^{\mathrm{e}}$ s. (Walter Scott, Charlotte M. Yonge).

Sont abordés des textes tels que The Court of Love, probablement du $\mathrm{Xv}^{\mathrm{e}} \mathrm{s}$., peu prisé par la critique mais que Ad Putter s'attache à valoriser en soulignant ses aspects ambivalents (à la fois subversifs et respectueux de la tradition courtoise) et l'érudition de son auteur, qui puisa notamment dans Charles d'Orléans, Le Roman de la Rose, Chaucer...

$\mathrm{Au}$ début du Xvi ${ }^{\mathrm{e}}$ s., Wynkyn de Worde publie The Squire of Low Degree, peut-être pour répondre aux attentes d'un lectorat bourgeois qui continuait d'apprécier les romans ou textes apparentés à ce genre et considérait aussi l'usage moral, didactique et politique que l'on pouvait faire des romans. Cette thèse a d'ailleurs été exposée par Janet Coleman dans l'ouvrage Medieval Readers and Writers, 13501400, (New York, Columbia University Press, 1981). Julia Boffey et Anthony Stockwell Garfiel d Edwards évaluent la dimension romanesque dans The Squire of Low Degree, qui ne réunit pas toutes les caractéristiques du roman traditionnel et semble suggérer que la valeur tient moins aux exploits accomplis qu'à l'ascension sociale. De plus, ces « romans » tardifs comme The Squire of Low Degree introduisent des changements formels (par ex., des dialogues) au détriment de l'action. Ces changements laissent penser qu'un certain type de roman parvint à échapper au déclin grâce à des modifications stylistiques et thématiques destinées à se concilier une clientèle nouvelle.

Andrew Lynch, enfin, examine le renouveau du roman - point d'orgue de cet ouvrage sur l'évolution du roman - à travers la lecture analytique de deux auteurs, Walter Scott et Charlotte M. Yonge, tous deux couvrant un empan temporel allant de la fin du XVIII ${ }^{\mathrm{e}}$ au tout début du XX⿳⺈冂大 $\mathrm{e}$., tous deux s'inspirant de romans de chevalerie (par ex. Ivanhoe ou Waverley pour W. Scott, The Heir of Redclyffe pour C. M. Yonge). Cependant, même si la renaissance médiévale put traduire une aspiration à revenir vers des valeurs morales propres à élever la jeunesse, l'époque représentée par W. Scott, puis C. M. Yonge, sut aussi dénoncer les dérives violentes de la chevalerie et adopter une lecture discriminante et nuancée du roman.

Les éditrices livrent un volume d'hommages aisé à lire, très documenté, et surtout très vivant car toutes les contributions semblent directement dialoguer avec $\mathrm{H}$. Cooper et ses nombreuses publications. L'ensemble est très cohérent. On note quelques reprises d'études déjà données ou publiées sans toutefois que ces reprises atténuent la force de la problématique de l'évolution littéraire d'un genre observée d'une manière complète tenant compte de l'adaptation des sources, des changements historiques et sociaux, des modifications des goûts du lectorat.

Martine YvERNAULT

EA 1087 - EHIC

Université de Limoges 